\title{
Effect of telehealth-to-home interventions on quality of life for individuals with depressive and anxiety disorders
}

This article was published in the following Dove Press journal:

Smart Homecare Technology and TeleHealth

7 November 2014

Number of times this article has been viewed

Logan Durland'

Alejandro Interian ${ }^{1,2}$

Ingrid Pretzer-Aboff ${ }^{3}$

Roseanne D Dobkin'

'Rutgers, The State University of New Jersey, Robert Wood Johnson Medical School, Department of Psychiatry, Piscataway, NJ, ${ }^{2}$ The New Jersey Healthcare System, Veteran Affairs Medical Center, Lyons, NJ, ${ }^{3}$ University of Delaware, School of Nursing and Biomechanics and Movement Sciences Program, Newark, DE, USA

Correspondence: Roseanne D Dobkin Rutgers, The State University of New Jersey, Robert Wood Johnson Medical School, Department of Psychiatry, 675 Hoes Lane, Room D-3 17, Piscataway, NJ, 08854 USA Email dobkinro@rutgers.edu
Abstract: Although millions of individuals suffer from mental health problems worldwide, only a small fraction receives adequate treatment. The high prevalence of depression and anxiety observed worldwide, in conjunction with very low rates of treatment utilization, are of great clinical significance, as these psychiatric conditions are two of the most important determinants of quality of life (QoL). Telehealth interventions have been touted as potential solutions to these mental health care disparities, with great interest and utility demonstrated across a diverse array of medical and psychiatric populations. Telehealth interventions may be clinic-based or homebased. The primary objective of this paper is to highlight the extent to which telehealth-to-home interventions positively impact multiple facets of QoL for individuals with depression and anxiety disorders, including those with comorbid medical conditions. While QoL outcomes are important to consider in any assessment of treatment effectiveness, QoL enhancement has received limited attention in the telemental health literature to date. All studies included in the present review evaluate telehealth-to-home treatments, assess QoL outcomes, and incorporate some degree of live, synchronous therapist-patient contact. Recommendations to advance the application of telehealth-to-home approaches are proposed and include: additional research on video-to-home telehealth platforms, strategies to increase the adoption of telehealth-to-home interventions amongst mental health treatment providers, national legislative reform to establish fair and appropriate reimbursement parameters for telehealth-to-home care, standardized definitions of telehealth-to-home services to facilitate provider engagement and reimbursement, and systematic approaches for measuring the cost-effectiveness of telehealth-to-home treatments.

Keywords: telehealth-to-home, quality of life, depression and anxiety disorders

\section{Introduction}

The term "telehealth" refers to health care information that is delivered through a variety of media (eg, email, web-based interactive platforms, telephone calls, self-help books), rather than through face-to-face meetings between patients and providers. ${ }^{1}$ In the context of mental health services, telehealth refers to assessment, psychoeducation, medication monitoring, and group or individual therapy conducted via these methods. Telehealth treatments are increasingly gaining attention in the clinical psychology repertoire, as relevant empirical evidence comes to light and the potential advantages of the telehealth approach in dealing with challenges currently facing the field become apparent. ${ }^{2}$ Telehealth is used to connect a patient in one location with a provider in a separate location. This can include linking a rural clinic with an urban medical center. It can also include linking a patient in their home (via telephone or videoconferencing) to a provider, which is referred to as "telehealth-to-home", and is the focus of the current paper. 
The rationale for telehealth interventions to treat psychological disorders rests on evidence of a vast disparity between the number of individuals in need of mental health services and the number who actually utilize them. Based on an international World Health Organization (WHO) study, roughly $50 \%$ of the US population will meet criteria for a psychological diagnosis at some point in their lifetime and the 12-month prevalence of diagnosable mental illness in the USA was estimated at $25 \%$, translating to approximately 75 million current US citizens. In a WHO-sponsored international study of more than 25 countries, the USA had the highest lifetime and 12-month psychological diagnosis rates. Moreover, significant mental health burden has been observed in numerous countries throughout the world. ${ }^{3}$

Depression and anxiety disorders are two of the most pervasive and destructive mental health issues facing the field of clinical psychology. The WHO reports that depression is the third most significant cause of illness worldwide, ${ }^{4}$ and that anxiety disorders are the most common class of disorders in the general population. ${ }^{3}$ The lifetime prevalence of major depression in US adults is $16 \%$, and the 12 -month prevalence rate has been calculated at $7 \%,{ }^{5}$ with many more individuals meeting criteria for subclinical depression or dysthymia. Moreover, the lifetime prevalence of anxiety disorders in US adults has been reported at $28.8 \%{ }^{6}$

\section{Mental health care disparities}

Although millions of individuals suffer from mental health problems worldwide, only a small fraction receives adequate treatment. Many do not go on to seek help and those who do initiate treatment often prematurely discontinue services. ${ }^{6}$ For example, one study found that only $10 \%-45 \%$ of patients with major depression ever schedule an initial appointment, ${ }^{7}$ while another research group demonstrated that, of those who do attend at least one appointment, more than half drop out of treatment. ${ }^{8}$ Individuals with chronic medical conditions may be among the most vulnerable to disparities in mental health care access and utilization. Specifically, estimates suggest that at least $50 \%$ of depressed medical and homebound elderly patients are suboptimally treated for their psychiatric concerns, with a significant proportion never receiving any mental health care. ${ }^{9-12}$

The mental health field is replete with barriers to seeking care that account for the treatment gap. These include a lack of financial resources, lack of readily available treatment, lack of time, lack of caregiver support with treatment use, transport difficulties, limited provider base in a given specialty area, the effects of physical impairment or psychological distress, stigma, cultural factors, and poor mental health literacy. ${ }^{11,13,14} \mathrm{~A}$ significant portion of these factors involves difficulty accessing mental health care services. First, treatment facilities are often too distant for many patients to reach. Services tend to be disproportionately concentrated in more densely populated urban areas, such that those living in rural areas are geographically isolated from care. ${ }^{15-17}$ For example, many primary care providers in rural settings report a dire need for mental health services, particularly the delivery of evidence-based treatment modalities. ${ }^{18}$ Second, access to mental health treatment for many individuals is limited by physical conditions that make leaving the home a significant challenge, ${ }^{11}$ independent of the distance to the treatment facility. Third, specialized knowledge and expertise is often needed to treat complex patient groups with multiple medical and psychiatric comorbidities, as well as those from diverse cultural and ethnic backgrounds. The number of providers with these specialized skill sets is likely limited in most geographic regions. ${ }^{11}$ Telehealth services, utilizing telephone or video-to-home platforms, hold promise for overcoming these access barriers.

\section{Impact on quality of life}

The term quality of life (QoL) broadly refers to an individual's general well being and the extent to which a person is able to enjoy the important possibilities that life has to offer, across multiple, heterogeneous domains (eg, physical, psychological, spiritual, social). ${ }^{19,20}$ For example, several specific subtypes of QoL have been proposed, and include areas such as engagement in activities of daily living, social functioning, community involvement, physical mobility, pain, emotional well-being, and fatigue. Related to this, health-related QoL refers to the psychosocial consequences of the physical, mental, and social aspects of a medical illness. ${ }^{21}$

The high rates of depression and anxiety observed worldwide, in conjunction with very low rates of treatment utilization, are of great clinical significance, as these psychiatric conditions are two of the most important determinants of QoL. Approximately $60 \%$ of individuals with major depressive disorder experience severe functional impairment. ${ }^{5}$ Moreover, research indicates that anxiety disorders adversely impact $\mathrm{QoL}$ at both the threshold and subthreshold severity levels. ${ }^{22}$ In medical populations, depression and anxiety exert a stronger influence on all aspects of QoL than physical disability. ${ }^{23}$ Thus, successful treatment of depression and anxiety is a necessary prerequisite to optimization of functioning, satisfaction, and enjoyment across all areas of life. In addition, understanding QoL outcomes is necessary to gauge whether treatments 
not only alleviate psychiatric symptoms, but also the daily life struggles associated with mental health concerns.

\section{Telemental health solution?}

Telehealth interventions have been touted as potential solutions to these mental health care disparities, ${ }^{24}$ with great interest and utility demonstrated across a diverse array of medical and psychiatric populations. Telehealth not only eliminates geographical barriers to evidence-based mental health treatment, but also permits the centralization of services, allowing for a concentration of expert providers to deliver specialty psychiatric care to individuals across great distances. Studies suggest that telehealth-to-home treatments for depression ${ }^{25}$ and anxiety ${ }^{26}$ are beneficial, with attrition rates substantially lower than those observed for in-person, face-to-face psychotherapy. ${ }^{25}$ For example, in a recent randomized controlled trial, telehealth-to-home, eg, telephone-based cognitive behavioral therapy (CBT), was associated with significant reductions in depressive symptoms, as well as improved treatment adherence (compared with in-person face-to-face CBT), in depressed primary care patients. ${ }^{25}$ In a separate study, telephone-based CBT was found to be more effective than usual care (ie, antidepressants) in the long-term treatment of depression in primary care. ${ }^{27}$ Notably, telephone management (symptom monitoring and support) of depressive symptoms, as well as telephone management plus telephone CBT, were associated with an additional 29 and 46 depression-free days, respectively, compared with clinic-based usual care, over 24 months. ${ }^{28}$ Finally, telemedicine-based collaborative care clinic models for depression in rural federally qualified health centers have been linked with significantly greater reductions in depression symptoms compared with practicebased collaborative care. ${ }^{29}$ Importantly, therapeutic alliance (eg, quality of the relationship between therapist and client) and adherence (eg, fidelity to evidence-based treatment protocols) remains high across a variety of telehealth treatment delivery methods. ${ }^{10,30,31}$

\section{Rationale for the focus of this paper}

To date, several comprehensive review papers have been published regarding the feasibility and effect of telemental health interventions for both medical and psychiatric disorders., ${ }^{2,-43}$ Common telehealth delivery methods include videoconference, telephone, Internet, or email. Primary therapeutic modalities include CBT (a structured treatment approach that targets the thoughts and behaviors related to the onset and maintenance of psychopathology), interpersonal psychotherapy (IPT; a manualized treatment in which depressive symptoms are addressed by seeking to reduce interpersonal conflict and stress), problem-solving therapy (a therapeutic approach for depression in older adults that seeks to increase effective use of social, ie, help-seeking and personal resources), ${ }^{44}$ supportive therapy, antidepressant medication management, and psychoeducation.

Telehealth interventions may be clinic-based or homebased. In the case of clinic-based telemedicine, experts provide remote specialty care to patients in the patients' local community health center. Clinic-based telemedicine, at a minimum, requires patients to travel from their homes to another location (eg, medical office). Alternatively, telehealth interventions delivered directly into the patients' home completely eliminate the need for patient travel. Both approaches have merit. The appropriateness of telehealth-to-home versus telehealth-to-clinic depends on a range of clinical variables, such as medical complexity, the need for ancillary medical staff to be on location during the clinical encounter, and the patient's comfort level with technology.

The primary objective of this paper is to highlight the extent to which telehealth-to-home interventions positively impact multiple facets of QoL, for individuals with depression and anxiety disorders. The clinical significance of this metric (eg, QoL enhancement), has only received limited attention in telemental health reviews to date. All studies included in the present review incorporate some degree of live, spontaneous therapist-patient contact. The rationale for these three key inclusion criteria (telehealth-to-home, QoL outcomes, live synchronous therapist-patient contact) is further delineated below.

\section{Telehealth-to-home}

One of the primary advantages of telehealth approaches over traditional face-to-face therapy is that they reduce barriers to treatment related to transport and geographic location. To date, several studies have explored the use of telehealth-toclinic interventions implemented in primary care settings, which are often the only available source of mental health treatment in rural settings. ${ }^{45-49}$ As only $25 \%$ of primary care settings have mental health professionals on staff, ${ }^{50}$ collaborative care approaches have been developed in which offsite mental health professionals interface with patients and primary care providers in the clinic using videoconferencing, telephone, and the Internet, with promising results obtained within the Veteran Affairs (VA) primary care system ${ }^{29,51}$ and elsewhere. While telehealth treatments such as these, based in primary care or other satellite locations, may increase access to mental health services in certain scenarios, eg, in rural 
areas where primary care services are locally available but mental health services are not, they still require the patient to secure transport to a clinic setting, which may be a significant challenge for patients struggling with severe physical, psychological or economic limitations. Therefore, our review emphasizes studies in which interventions have been delivered directly to patients' homes, typically via telephone or videoconference technology.

\section{Quality of life}

While many studies of psychological interventions focus on their impact on symptom frequency and severity, symptom reduction that corresponds to a measurable improvement in QoL is of the greatest clinical significance. The use of QoL measures in assessing patient outcomes improves the construct validity of "treatment success" and bypasses the danger that symptom severity and QoL are uncorrelated. With that perspective in mind, this review focuses on telehealth studies that include a diverse array of QoL measures in addition to symptom severity assessment.

\section{Synchronous patient-therapist contact}

One important criterion differentiating telehealth approaches is the degree to which they offer real-time contact between patient and provider. On the one end of the spectrum are treatments delivered entirely or largely via interactive software programs and the Internet. ${ }^{33,38-43,52,53}$ Many such treatments utilize a CBT approach, perhaps in part because the emphasis of CBT on the collection and analysis of data relating to patients' thoughts, feelings, and behaviors is well suited to a computerized treatment setting. While many telehealth interventions entail minimal patient-provider contact (eg, automated, interactive, computerized self-help programs), others require a level of contact comparable with that of traditional face-to-face psychotherapy (eg, telephone or videoconferencing-based interventions).

Several large-scale, randomized controlled trials of asynchronous computerized interventions (eg, "Beating the Blues" or "MoodGYM") have yielded promising results with respect to depression, anxiety, and QoL. ${ }^{42,54-57}$ However, varying levels of therapist contact offer different advantages. Low-contact telehealth interventions are by nature more scalable and cost-effective than high-contact therapies. Yet, several studies suggest that telehealth interventions with greater contact lead to better patient outcomes. ${ }^{58}$

A meta-analysis by Andersson and Cuijpers ${ }^{33}$ showed that interventions with some professional support yielded a significantly greater outcome (Cohen's d=0.61) than those without professional help (Cohen's $d=0.25$ ). In a separate meta-analysis of 12 randomized controlled trials using Internet-based CBT interventions, with contact protocols ranging from no therapist contact at all to indirect monitoring and feedback with supplemental telephone check-ins, a large effect size was found in interventions in which there was at least some therapist support, and a small effect size was found for interventions in which there was no therapist support. ${ }^{43}$ Moreover, a study comparing the MoodGYM computerized intervention alone and in combination with face-to-face CBT showed that while the computerized intervention alone was not significantly more effective than a no-treatment control, MoodGYM in combination with face-to-face CBT was more effective than either active intervention alone. ${ }^{59}$

Although drawn from a relatively small number of studies with a modest amount of contact protocol heterogeneity, these findings suggest that despite the advantages of automating interventions, the involvement of a real human being in the therapeutic process remains important. The association between greater therapist contact and outcome may be due to common factors associated with psychotherapy outcomes, such as rapport, empathy, and warmth. In addition, therapist contact allows for greater ease in individually tailoring treatment in high-contact therapies. Based on these results and the thorough summaries of low-contact interventions already available in the literature, ${ }^{33,38,39}$ this review focuses on synchronous, moderate-contact, and high-contact interventions.

Representative articles that meet the aforementioned study selection criteria are further described below for those with psychiatric and medical comorbidities, as well as for primary depressive and anxiety disorder disturbances.

\section{Study selection methods}

In identifying studies eligible for inclusion in the paper, we first conducted several literature searches within the PsychInfo database. Searches using combinations of the search terms "telemedicine", "telehealth", "depression", "major depression", and "anxiety" yielded approximately 250 studies. A second search using combinations of the search terms "review", "telehealth", "telemedicine", "anxiety", and "depression" yielded approximately 45 review articles. Empirical studies that met the three study selection criteria described above, and reviews that referenced studies that met these criteria, became candidates for inclusion. Within this set of articles, we preferentially selected articles that were more methodologically sound (eg, larger sample 
size, active controls) or added unique content (eg, illustrated telehealth interventions with different population). In addition, approximately 40 articles relating to disorder prevalence and other supporting data were gathered, and a smaller subset of articles was gleaned based on the currency and relevance of the articles. The final set of empirical studies, reviews, and supporting articles totaled 125 .

\section{Review of the evidence Medical populations}

A considerable body of research has addressed synchronous telemedicine interventions for patients with comorbid medical and psychiatric conditions. Telehealth-to-home treatments (predominantly telephone-based) have targeted depression and anxiety in the context of chronic medical conditions such as multiple sclerosis, ${ }^{60-70}$ Parkinson's disease, ${ }^{10,71} \mathrm{HIV} / \mathrm{AIDS},{ }^{72-74}$ diabetes, ${ }^{75,76}$ heart disease, ${ }^{76,77}$ traumatic brain injury, ${ }^{78,79}$ dementia, ${ }^{80-82}$ hematopoietic stem cell transplantation, ${ }^{83}$ spinal cord injury, ${ }^{65}$ amputation, ${ }^{65}$ and stroke, ${ }^{65,84}$ among others. For example, Dobkin et $\mathrm{al}^{10}$ found that ten sessions of telephone-based CBT were associated with notable improvements in depression, anxiety, and proactive coping for depressed people with Parkinson's disease in a treatment development pilot, with larger controlled studies under development.

Medical illness increases the likelihood that patients will confront significant mobility barriers to mental health treatment, making populations with medical and psychiatric comorbidities particularly appropriate for telehealth-to-home approaches. Importantly, in our experience, studies of patients with comorbid medical and psychiatric conditions are more likely to include QoL outcome measures than studies focusing on a single mental health diagnosis, since in the former, the psychological concerns are being considered in the wider context of the patients' general health, making studies on populations with chronic medical conditions particularly relevant to this review.

In general, research supports the feasibility and effectiveness of telehealth-to-home treatments for medically ill populations with comorbid depression and anxiety that combine traditional therapeutic approaches with interventions specifically targeting medical symptoms. However, one noteworthy caveat to telehealth research in such populations is that medical treatment is often delivered concurrently with mental health treatment, making it more difficult to separate the effects of the study intervention from those of medical treatment. Representative studies are discussed further below.

\section{Multiple sclerosis}

Several randomized-controlled trials have demonstrated the efficacy of telephone-administered treatments in alleviating depression associated with multiple sclerosis. ${ }^{60-70}$ For example, a study by Mohr et $\mathrm{al}^{70}$ compared the effectiveness of telephone-mediated CBT with telephone-mediated emotionfocused therapy in treating 127 participants suffering from depression and multiple sclerosis. The central goal of the study was to assess whether a CBT treatment administered by telephone conferred benefits beyond those attributed to nonspecific factors (eg, the therapeutic relationship, empathy, personalized treatment based on a manual) putatively at play in emotion-focused treatment. Each treatment involved weekly 50-minute sessions over 16 weeks. Care was taken to isolate the effects of treatment content specific to CBT, in order to determine if concrete coping skills could be taught effectively through this remote platform. Study results showed significant reductions in depressive symptoms and frequency of a major depressive disorder diagnosis for both emotion-focused and CBT treatment groups. However, at post-treatment, patients receiving CBT exhibited even greater decreases in depressive symptoms and rate of major depressive disorder diagnoses, and greater increases in positive affect, compared with patients receiving emotion-focused therapy. Telephone-based treatment was also associated with significant improvements in overall QoL, social support, and coping skills, as well as reductions in multiple sclerosisrelated physical disability, fatigue, and pain. ${ }^{61,62}$ Treatment gains were maintained at follow-up for both groups.

In a separate study ${ }^{63}$ by the same group that compared telephone-based CBT with usual care for individuals dealing with multiple sclerosis and depression, telephone-based CBT was not only associated with significant reductions in depression, but was also linked to improved adherence to disease-modifying therapy, interferon $\beta$-1a, a critical functional outcome for those living with multiple sclerosis. Telephone-based physical activity counseling, utilizing a motivational interviewing approach, has also been found to reduce both the severity and overall incidence of major depressive disorder in multiple sclerosis, to improve physical activity levels, and to reduce the physical side effects associated with having multiple sclerosis. ${ }^{64}$

\section{Diabetes and cardiac health}

Noteworthy telehealth-to-home platforms have also been established for treating depression in diabetic and cardiac patients. Piette et al compared the effectiveness of a telephone-delivered CBT treatment, combined with a walking 
program, with a usual care control (provision of self-help materials and psychoeducation) for 291 patients suffering from diabetes and depressive symptoms. ${ }^{75}$ The experimental treatment consisted of 12 weekly telephone-administered treatment sessions followed by nine monthly booster sessions, in which depressive symptoms were addressed first, followed by the importance of the walking program. Relative to the control group, the treatment group showed a significant improvement in depressive symptoms (remittance rate: treatment group 58\%, control group 39\%), systolic blood pressure, and physical activity during treatment, as measured by steps taken per day. Physical functioning, role limitations, perceived coping skills, and self-efficacy regarding physical activity improved only in the treatment group. Unfortunately, however, the study results did not show a significant improvement in glycemic control for the treatment condition, which may have been the result of a floor effect, since $70 \%$ of patients showed plasma glucose concentrations below $8 \%$, within $1 \%$ of the recommended range for diabetic plasma glucose. ${ }^{85}$ Thus, although this treatment condition did not confer an advantage over the control on a main health-related measure in the diabetic population (glycemic control), relative improvements in physical activity and a number of other QoL dimensions were observed, as well as a significant higher remission rate for depression.

A collaborative care program for 214 individuals with depression and diabetes or heart disease, with an innovative telehealth follow-up component, also yielded clinically significant effects on key psychiatric, medical, and QoL indicators. ${ }^{76}$ Participants were randomly assigned to receive either standard medical treatment (in primary care clinics) or guideline-based collaborative care management, intended to simultaneously manage risk factors for multiple diseases. The 12-month experimental intervention emphasized self-care approaches, motivational interviewing, problem-solving, and pharmacotherapy. The program encompassed ten officebased treatment sessions (primary care), followed by ten telephone-delivered maintenance sessions. The collaborative care management intervention was associated with significant improvements in key medical indicators for diabetes and cholesterol (eg, hemoglobin, blood pressure, cholesterol), depression, QoL, global functioning, and satisfaction with depression treatment.

Lastly, a large study compared a telephone-based psychiatric intervention with the control condition among 302 depressed patients in surgery recovery from a coronary bypass artery graft. ${ }^{.7}$ The treatment condition consisted of medication management, psychoeducation around depression, discussion of future treatment options, and referral to individual psychotherapy in the community, if requested. Participants in the usual care condition were informed of their depression status, but were not provided with any treatment. Changes in depressive symptoms were assessed with the 17-item Hamilton Depression Rating Scale, general mental and physical well-being was assessed with the Mental and Physical Health subscales of the Short Form-36 Health Survey, and physical functioning were assessed with the Duke Activity Status Index. The treatment group demonstrated significantly greater improvements compared with the usual care group on depression, mental well-being, and physical functioning (Duke Activity Status Index).

\section{HIVIAIDS}

Several telehealth-to-home approaches have successfully addressed depression in HIV-positive patients. One such study compared usual primary care with telephone-delivered interpersonal therapy for 79 rural HIV-positive patients suffering from depression..$^{72}$ The usual care condition involved unrestricted access to resources provided by local AIDS service organizations and individual therapy in the community. Depressive symptoms were assessed using the Beck Depression Inventory-II, and QoL indices (eg, relational health and social role performance) were measured using the UCLA Loneliness Scale, Outcome Questionnaire-45, and Provision of Social Relations Scale. Over a 6-week trial period, participants in the IPT condition experienced clinically significant improvement in both depression symptoms and QoL, as assessed by the measures above, relative to the control group. Although the depression response rate for the treatment condition was modest $(23 \%$ for treatment condition versus $8 \%$ for control condition), it compared favorably with response rates typically seen in effectiveness trials. ${ }^{86}$ In addition, the study's treatment length was typical of community mental health settings, suggesting that it may have strong external validity, although IPT treatment for depressed adults typically spans $12-16$ weeks, ${ }^{87}$ and treatment results might have been improved with added sessions.

Additional studies in HIV-positive samples have also found telephone-based treatment approaches, including CBT, to improve both depression and adherence to antiretroviral medication, and to reduce sexual risk-taking behavior. ${ }^{73,74}$ For example, Himelhoch et al compared telephone-based and face-to-face CBT for depression in 34 low-income individuals living with HIV/AIDS. ${ }^{73}$ The intervention involved eleven sessions delivered over 14 weeks, and included a treatment workbook provided to patients and a therapist manual for 
clinicians. Both groups showed significant reductions in depressive symptoms (Cohen's d effect size estimates on all depression measures were 0.86 or greater for both groups) and reported a strong working alliance and high satisfaction with treatment. Not only was telephone-administered therapy just as effective as in-person treatment, but the telephone-based CBT group also showed significantly greater medication adherence at post-treatment than the face-to-face group. These results suggest that telephone-based interventions may be a feasible, cost-effective means for helping low-income urban residents with depression and chronic comorbid medical illness, which is especially encouraging considering the evidence that economic disadvantage is often a strong barrier to treatment.

\section{Caregiving}

Telehealth, in addition to being utilized in the treatment of medically ill patients, has been implemented to address depression in caregivers of those with chronic medical conditions. Telephone-administered CBT has been shown to be more effective in reducing psychological distress and burden, compared with routine education and support, for distressed rural caregivers of individuals with dementia. ${ }^{82}$ Telephone-based support groups for dementia caregivers have also been linked with decreased depression for older (and possibly more isolated) care partners, as well as increased perception of "personal gains". ${ }^{80}$ The concept of "personal gains" tapped into several QoL indicators for caregivers, such as the ability to form new friendships, strategies for handling loneliness, stress, and depression, as well as techniques for mobilizing health care resources and dealing with family relationships.

Most recently, Glueckauf et al compared face-to-face and telephone-based CBT interventions for 14 depressed AfricanAmerican caregivers of dementia patients. ${ }^{81}$ The intervention involved seven group sessions devoted to dementia psychoeducation and CBT skill development (eg, assertiveness, problem-solving, cognitive restructuring), as well as five one-to-one sessions, in which individual problem-solving and goal-setting was addressed. At post-treatment, significant improvement in symptoms of depression, support assistance, and subjective burden were found in both conditions, with no significant differences in physical symptoms reported. Notably, effect sizes for QoL indicators (assistance support and subjective burden) were in the moderate range. A larger controlled trial is currently underway.

\section{General depression}

Although the evidence-base supporting the use of telehealth technologies to treat primary depressive disorders is quite impressive, ${ }^{25,29,34,35,54,88-93}$ the overwhelming majority of these seminal studies did not meet the inclusion criteria for the current review (eg, telehealth-to-home, focus on QoL outcomes, synchronous therapist-patient contact). A significant number of these "primary depression" trials (ie, not involving populations with medical comorbidities) do not assess QoL outcomes, employ a telehealth-to-clinic protocol, or have very limited or no therapist contact. Thus, many recent and well designed studies are not included for this reason. ${ }^{25,29,92,93}$ Only representative studies that met our inclusion criteria are detailed below.

Interactive videoconferencing to home, using standard webcams and Internet equipment, has effectively been used to treat depression in underserved populations. In a study conducted by Moreno et al, 167 depressed adults from a community-based clinic were randomly assigned to receive a monthly webcam treatment intervention, administered by a psychiatrist, or to usual care by their primary care provider in a primary care clinic. ${ }^{94}$ The videoconference intervention was superior to usual care by a primary care provider, as measured by reductions in depressive symptoms and improvement on two QoL measures assessing enjoyment/satisfaction with life and level of perceived disability. Notably, the treatment population was exclusively Hispanic, a group with substantially lower rates of mental health treatment utilization compared with non-Hispanic whites. ${ }^{95}$ The acceptability and effectiveness of telepsychiatric interventions in the Moreno study illustrates the promise of telehealth for expanding mental health treatment access to underserved populations.

In another study, nurses in a primary care setting with limited experience in mental health treatment were briefly trained to implement management, of antidepressant medication, depression psychoeducation, and behavioral activation, and were then assigned to treat 102 patients with depressive disorders via telephone over a 6-month period. ${ }^{96}$ Depression symptoms were measured using the Hamilton Depression Rating Scale. Fulfillment of work roles was assessed with the Work Limitations Questionnaire, mental and physical health was assessed using the Short Form-12 Mental and Physical Composites, and enjoyment of free time and domestic functioning were also assessed. At follow-up, patients demonstrated significant improvement in depressive symptomatology, with $63 \%$ of patients exhibiting a $50 \%$ or greater reduction in Hamilton Depression Rating Scale scores. In addition, patients showed significant improvements in the Work Limitations Questionnaire total score (baseline mean 70.4; 6-month assessment mean 87.2), and improvements in all subscales of this questionnaire (eg, time, physical, mental, 
and interpersonal demands) compared with baseline. Based on estimations of the amount of work productivity associated with a given gain in the Work Limitations Questionnaire score and the professional occupations of the sample, it was calculated that the intervention saved roughly $\$ 2,600$ of productivity per patient per year. Although this study did not include a control condition, and its treatment condition makes it difficult to determine the relative contributions of each therapeutic component (talk therapy, antidepressant medication) to treatment outcome, the combined psychosocial/ psychiatric intervention effectively simulates "real world" treatment, and the significant improvements across depression and QoL measures suggest that telephone-mediated psychiatric support may be effective for treating unipolar depression.

Lastly, a recent study piloted a telephone-based adaptation of problem-solving therapy (PST) in primary care, randomizing 121 depressed older adults to an in-person PST group, a telephone-based PST group, or a telephone-based control group in which therapists provided supportive therapy and symptom monitoring. ${ }^{97}$ Sessions for all conditions were delivered weekly for 6 weeks, and sessions for both PST condition were 60 minutes long, while the control group telephone sessions were 30 minutes long.

Analyses showed significant advantages in depressive symptom reduction for the in-person PST (Cohen's d=0.45) and telephone PST (Cohen's $\mathrm{d}=0.55$ ) compared with the control group. At 24-week follow-up, the 50\% symptom reduction rate was $48.5 \%$ for telephone-PST, $42.4 \%$ for inperson PST, and $27.6 \%$ for the control group. There was a significant inverse relationship between depression severity and personal resourcefulness at the 12-week and 24-week follow-up points. While analyses did not indicate a mediating effect of resourcefulness in depressive symptom reduction for any of the treatment conditions, the authors hypothesize that this QoL indicator (resourcefulness) increased for all study participants due to improved perceptions of self-control and self-efficacy that resulted from trial participation. Overall, the results suggest that that the problem-solving therapy delivered via telehealth may be a highly effective treatment for depression in older adults.

\section{Anxiety}

\section{Generalized anxiety disorder}

Brenes et al compared telephone-administered CBT with a psychoeducation control condition in treating a sample of 60 older adults (aged $>60$ years) suffering from generalized anxiety disorder, panic disorder, or anxiety disorder not otherwise specified. ${ }^{26}$ This intervention entailed telephone sessions in combination with a patient workbook covering topics such as relaxation, behavioral activation, cognitive restructuring, in vivo exposure, relapse prevention, and insomnia. Patients were assigned new chapters once they had adequately completed the previous chapter, as assessed by their clinician. Patients receiving telephone-administered CBT showed significantly greater reductions in anxiety symptoms, insomnia, anxiety sensitivity, and worry compared with controls at post-treatment, but only the gains in worry were maintained at 6-month follow-up. The telephone-administered CBT group also demonstrated significantly greater improvements in QoL (eg, mental well being) at 6-month follow-up. These data suggest that telephone-administered CBT can be effective in treating anxiety and improving QoL, but lasting recovery may require further sessions.

\section{Social phobia}

Some interventions have combined face-to-face and indirect telehealth-to-home contact in an effort to pool the benefits of each modality. For example, one study involving 64 patients suffering from social phobia compared a waitlist control with a 9-week, Internet-mediated treatment with email contact and two face-to-face group exposure sessions. ${ }^{98}$ Access to the next module in the program was contingent on patients' adequate completion of the homework assignment from the previous module, as assessed by their assigned clinician. Outcome measures included social phobia and depressive symptoms, along with happiness, meaning, and life satisfaction. Participants in the treatment condition showed significant improvement on measures of social anxiety, depression, and global QoL. The authors touted Internetbased therapy as advantageous in the treatment of social phobia due to its increased anonymity, but also decided that live exposure might be necessary for treatment impact, based on the absence of fully remote telehealth treatments for social phobia in the literature.

\section{Panic disorder}

For panic disorder, most telehealth treatments that are accessible from participant's homes emphasize Internet or telephone-based therapies. ${ }^{99}$ One study randomized participants to bibliotherapy augmented with therapist telephone guidance or four sessions of face-to-face group therapy. Both groups showed improved symptoms and reduced functional impairment. ${ }^{100}$ The study also reported that those randomized to the telephone guidance group were more likely to achieve high-end functioning (ie, minimal symptoms and zero panic 
attacks), relative to those assigned to the face-to-face group. Similarly, another study randomized participants to one of two therapist-guided, Internet-based CBT interventions for panic disorder (ie, one with and one without an exposure component). ${ }^{101}$ Both conditions yielded improved symptoms, as well as enhanced work and social functioning. Finally, a $77 \%$ remission rate for panic symptoms and improvements in avoidance and overall QoL were obtained (versus a waitlist control) for those participating in Internet-based CBT, supplemented by brief weekly telephone calls with a study clinician. ${ }^{53}$ In this trial, clients were required to turn in practice assignments before proceeding to the next module, a useful technique for encouraging treatment engagement, and only 3.9 therapist hours were spent per client over the 10 -week treatment. It should be noted that the literature for panic disorder treatments has a well developed evidence base for Internetbased therapies that are self-guided and include only minimal therapist support via email; ${ }^{102-105}$ such studies are beyond the scope of this review, as they do not meet inclusion criteria for synchronous patient-client contact.

\section{Post traumatic stress disorder}

Similar to major depression, research on telehealth-based psychotherapies for treating post traumatic stress disorder (PTSD) has been burgeoning. However, much of this work has focused on clinic-based telehealth therapies (and is thus beyond the scope of this review). This body of research generally shows that psychotherapy delivered via clinic-based telehealth helps achieve meaningful symptom reductions. ${ }^{106,107}$ On the question of equivalency to face-to-face treatments, the evidence is more mixed, and it is likely that studies powered to detect noninferiority are still needed.

The evidence base for PTSD therapies delivered via telehealth to patients' homes that capture QoL outcomes is much smaller. One study examined a home-based telehealth therapy for veterans with PTSD. ${ }^{108}$ The intervention utilized a combination of face-to-face, telephone, and Internet-based elements. Outcomes showed that $30 \%$ of recipients achieved high-end functioning, in terms of reducing symptoms to minimal levels. Another study compared the effectiveness of videoconference and face-to-face group CBT treatment for 38 veterans suffering from PTSD. ${ }^{109}$ Each treatment was 14 weeks long and consisted of 90-minute group therapy sessions. Outcome was assessed at pretreatment, post-treatment, and 3-month follow-up. PTSD symptoms, incidence of traumatic event exposure, and depressive symptoms were assessed, and patients were also asked to rate the frequency of social activities and the quality of their interpersonal relationships on a $1-10$ scale. A noninferiority design demonstrated that the face-to-face group outperformed the videoconference treatment, but the differences were not significant. Unfortunately, limited gains were exhibited by both groups.

One case study has also been published, and is notable given that it focuses on the use of home-based telehealth to assist a PTSD patient dealing with suicidality. ${ }^{110}$ Such cases address the common safety concern pertaining to homebased telehealth, in this case illustrating the successful use of telehealth to conduct a crisis intervention and the process by which it was achieved. Underway is a noninferiority, randomized trial examining prolonged exposure, delivery home-based telehealth versus face-to-face. ${ }^{107}$

\section{Summary}

Under the traditional, individual face-to-face treatment paradigm, mental health care is currently suffering from a "person-power" problem ${ }^{111}$ in which individual providers' caseloads are too small to close the gap between need and service availability..$^{90,112}$ Individual face-to-face psychotherapy delivered by mental health professionals with post-graduate training arguably has little hope of delivering treatment on the scale required, and alternative methods of treatments are needed. Telehealth-to-home treatments hold significant promise for reducing mental health care disparities and improving the many facets of QoL for individuals with depressive and anxiety disorders, including the elderly and those with chronic medical conditions (Table 1).

Telehealth-to-home approaches may be particularly advantageous for those with medical and psychiatric comorbidities. Several conclusions may be drawn from the telehealth-to-home research that has been conducted in medical groups to date. First, results suggest that telephone-mediated psychosocial interventions are helpful for depressed medical populations confronting significant barriers to face-to-face treatment. Second, therapeutic approaches that emphasize nonspecific factors (such as emotion-focused therapy) positively impact depression. Third, CBT for depression may offer benefits for QoL above and beyond those conferred by nonspecific therapeutic factors. Lastly, skills-based CBT can effectively be disseminated via a remote telehealth-to-home platform. Table 2 provides a quantitative summary of the evidence reviewed in this paper suggesting that telehealth interventions can offer benefits across a wide variety of QoL domains.

To date, a common concern with telehealth interventions is that the lack of the "human touch" resulting from remote contact might compromise the attractiveness of these 
Table I Key components of quality of life influenced by telehealthto-home treatment

\begin{tabular}{ll}
\hline $\begin{array}{l}\text { Global quality of life } \\
\text { Physical well-being }\end{array}$ & Physical functioning \\
& Physical role limitations \\
& Fatigue \\
& Pain \\
& Physical activity levels \\
& Adherence to disease-modifying therapies \\
& Physical complications of medical illness \\
& Enjoyment and satisfaction with life \\
& Personal resourcefulness \\
& Global assessment of functioning \\
& Perceived disabilities \\
& High end functioning (eg, near complete or \\
& I00\% resolution of psychiatric symptoms) \\
& Burden \\
& Satisfaction with mental health treatment \\
& Personal gains \\
& Work productivity \\
& Work limitations \\
& Loneliness \\
Social and occupational & Social role functioning \\
functioning & Relationship health and quality \\
& Assistance support \\
\hline
\end{tabular}

treatments to clients, since the strength of the client-therapist relationship has been cited as a powerful ingredient in therapeutic change. ${ }^{113}$ However, research on telehealth-tohome interventions for depression and anxiety show that these interventions have equivalent or even lower attrition rates than face-to-face individual psychotherapy, ${ }^{93,114}$ suggesting that such treatments may be just as acceptable as face-to-face therapy to clients, perhaps in large part due to their convenience. Suggestions to accelerate this promising line of research are further described below (Table 3).

The conclusiveness of this telehealth literature search may be limited by publication bias. First, the search terms used were relatively limited, and the search strategies used were simplistic. In addition, successful identification of articles depended on authors conceiving their work as "telemedicine" or "telehealth". Although these terms were encountered in many articles, studies that met our criteria but were not characterized as "telehealth" or "telemedicine" studies may have been missed by our searches. Third, although anxiety and depressive disorders are two of the most significant mental health challenges facing the field of psychology today, this paper's exclusive focus on these disorders limits the generalizability of findings regarding psychosocial telehealth interventions for other psychological problems. Lastly, papers that were not written in English were not considered, further limiting the pool of identifiable studies.

\section{Future directions \\ Additional research on video-to-home telehealth platforms}

Future research on all telehealth-to-home treatment modalities, with live synchronous therapist-patient contact is required, in order to accommodate a diverse array of clinical needs and patient preferences. To date, the majority of telehealth-to-home approaches have employed telephone or Internet-based platforms. Video-to-home treatments are just emerging, with exciting research in this area currently underway. For example, the VA Healthcare System in the USA is leading several promising innovations in telehealth. While several successful clinic-based VA telemental health programs have previously been launched in the areas of chronic pain, bipolar disorder, insomnia, and psychogenic seizures, amongst others, the VA is now in the process of expanding its rollout of video-to-home videoconferencing for mental health services. This will allow veterans to access care via their home computers and will eliminate the need to travel to satellite clinics. Several VA policies are expected to increase the pace of telehealth service uptake. For example, to incentivize more people to utilize home telemedicine services, last year the VA removed copays for people who see a physician via telemedicine in their homes. Also underway are large-scale, noninferiority trials comparing evidence-based treatments delivered via video-to-home with in-person treatment. For example, Gros et a ${ }^{107}$ report on an ongoing trial examining prolonged exposure therapy for veterans with PTSD, comparing video-to-home and inperson delivery. As studies like these accrue, the field will have an evidence-base for a delivery mode that completely eliminates geographical barriers and allows instantaneous exchange of verbal and nonverbal communication between patients and providers.

\section{Increasing uptake and implementation of telehealth-to-home interventions amongst mental health treatment providers}

Additional research on strategies to increase uptake and implementation of telehealth-to-home interventions amongst mental health treatment providers may prove beneficial. Telehealth-to-home interventions will radically transform the delivery of mental health services, marking a significant departure from the traditional model of office-based, faceto-face services. Accordingly, attitudes toward telehealth treatments among mental health providers are mixed, despite 
Table 2 Effect sizes for quality of life outcome measures across telehealth interventions

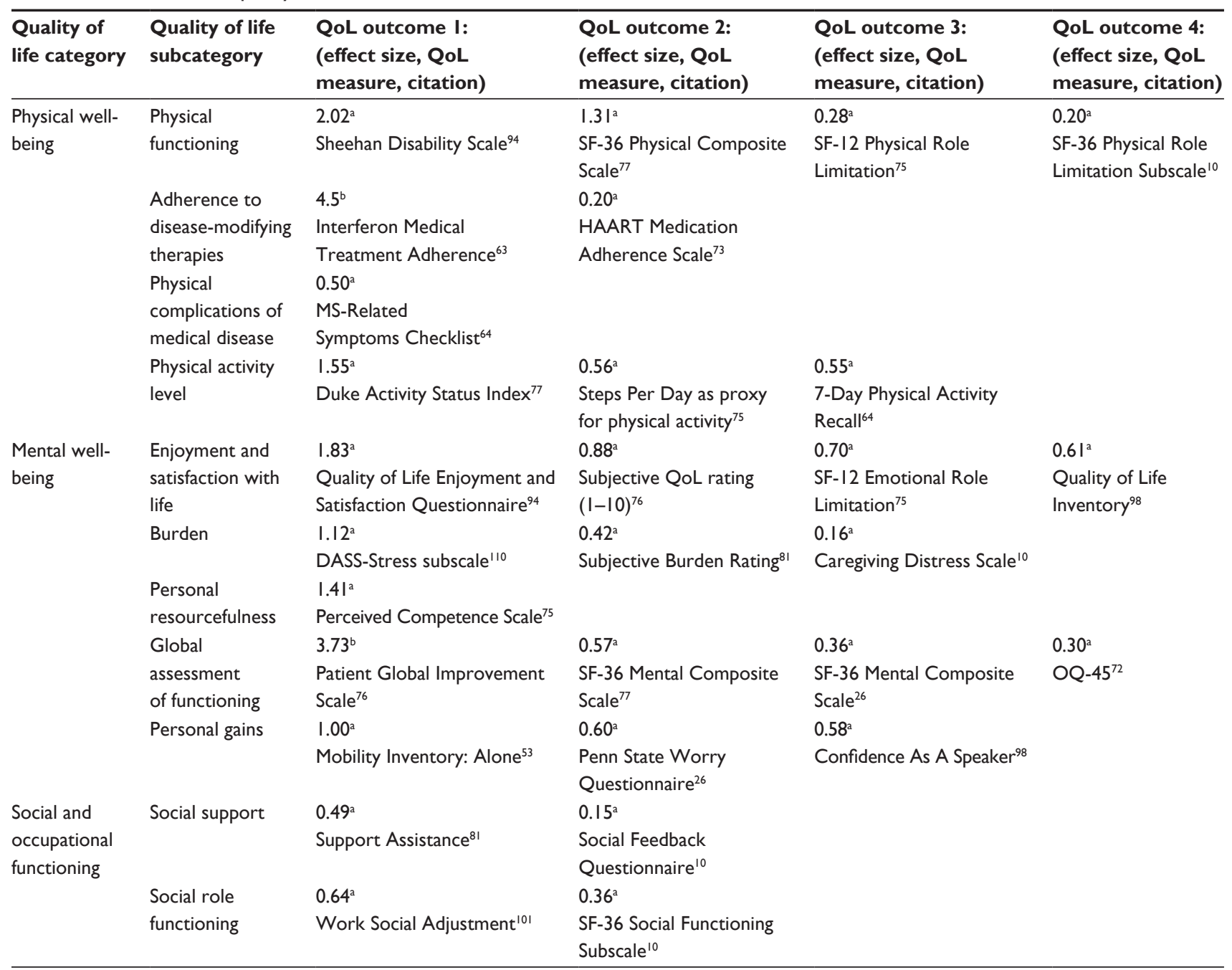

Notes: This table includes effects size measures from all studies highlighted in the text, where data for effect size calculations were available; andicates a Cohen's $d$ effect size; bindicates an odds ratio effect size.

Abbreviations: HAART, highly active antiretroviral therapy; SF, Short Form; OQ, Outcome Questionnaire; DASS, Depression Anxiety and Stress Scale; QoL, quality of life.

theoretical and empirical promise. A survey of Norwegian clinicians' views on the use of the Internet in therapy indicated that many were ambivalent and a minority had negative perspectives. ${ }^{115}$ Furthermore, therapists specializing in CBT were more likely to feel positively towards the use of the

Table 3 Recommendations to advance application of telehealthto-home treatment

I. Additional research on video-to-home telehealth platforms

2. Strategies to increase uptake and implementation of telehealth-tohome interventions amongst mental health treatment providers

3. National legislative reform to establish fair and appropriate reimbursement parameters for telemental health-to-home care

4. Standardized definitions of telehealth-to-home services to facilitate provider engagement and reimbursement

5. Systematic approaches for measuring cost-effectiveness of telehealthto-home treatments
Internet in therapy than those working in a psychodynamic mode. ${ }^{116}$ This finding may be related to the emphasis placed on the therapeutic relationship in psychodynamic circles, ${ }^{113}$ and the supposition that the development of this relationship requires face-to-face contact.

Additionally, in one trial, the authors reported that therapists initially reported fears about videoconferencing that seemed allayed by increasing experience with the technology. ${ }^{117}$ Other studies reveal provider apprehension about videoconferencing-based telehealth with regard to problems with nonverbal cues, alliance, and technical difficulties. ${ }^{118,119}$ Some evidence also suggests that providers may have greater concerns about alliance with this technology than patients. ${ }^{120}$ As the field examines the transformative potential of telehealth, the provider perspective will be critical, and providers are best viewed as 
stakeholders. The field can be best responsive to providers by understanding the nature of their discomfort and carrying out research that addresses the questions raised by these concerns.

\section{National, legislative reform to establish fair and appropriate reimbursement parameters for telemental health- to-home services}

At the current time, in the USA, telemental health-to-home services are reimbursed by third party payers under a very narrow set of circumstances, if at all. Regulatory and reimbursement policies vary considerably across states and are constantly evolving. ${ }^{121}$ Thus, regulatory and reimbursement factors presently do not generalize across institutions in the USA. Education, as well as legislative and regulatory reform, is needed to increase reimbursement for telehealth services, to the level that is obtained for the provision of in-person treatment. Importantly, telehealth-to-home does not offer less, and in many cases offers more than in person treatment, for those facing major barriers to mental health care utilization.

\section{Standardized definitions of telehealth- to-home care to facilitate provider engagement and reimbursement}

This definition should not be limited to one type of delivery platform. For example, both telephone and videoconferencing-to-home interventions hold great promise and require levels of time, skill, and expertise comparable with in-person treatment, and should be reimbursed as such. It is in the best interests of those served when the treatment delivery platform is dictated by clinical need and patient preference versus regulatory restrictions.

\section{Systematic approaches for measuring cost-effectiveness of telehealth- to-home interventions}

To date, there is no systematic strategy for determining the cost-benefit of telehealth interventions, and few studies have monitored cost-effectiveness as an outcome. Some studies have tracked the number of depression-free days (although there is no universally accepted definition as to what this is worth in dollars or QoL), as well as the decrease in need for inpatient medical services over time. For example, Godleski et al $^{122}$ found that clinic-based videoconferencing resulted in a $25 \%$ decrease in inpatient psychiatric hospitalizations over a 4-year period. Other research groups have confirmed similar findings. ${ }^{123}$

Cost-effectiveness has also been measured by comparing interventions based on the amount of clinical improvement per dollar invested in care, either by the patient, the health care system, or both. A study that compared videoconference and face-to-face psychiatric depression treatments for 495 rural patients in Canada showed that clinical outcomes for the two interventions were comparable, and that the telehealth intervention was roughly $10 \%$ less expensive to implement, ${ }^{123}$ suggesting a cost-effectiveness advantage for telehealth by the metric described above. Although this was not a telehealth-to-home study since the videoconference treatment was delivered at a primary care facility, its cost-effectiveness metric could be applied to telehealth-tohome interventions. Furthermore, a major source of cost inefficiency for mental health services pertains to missed appointments. Missed appointments leave agencies paying for operational costs for time slots that remain unfilled. The potential for telehealth-to-home to reduce missed appointments therefore may help improve this inefficiency. Studies addressing this question will likely provide much needed policy information for mental health administrators.

Prospective longitudinal studies capturing a diverse array of service utilization parameters, including referrals for laboratory work and other diagnostic tests, ${ }^{124}$ are required to more comprehensively address issues of cost-effectiveness. Costs associated with both medical and psychiatric service utilization should be captured, given that suboptimally treated mental health conditions significantly increase the risk of physical and cognitive deterioration. Unique cost-effectiveness paradigms are likely to be required for screening, prevention, and intervention services, as well as for synchronous and asynchronous models of treatment delivery.

\section{Disclosure}

The authors report no conflicts of interest in this work.

\section{References}

1. American Telemedicine Association. What is telemedicine? Available from: http://www.americantelemed.org/about-telemedicine/what-istelemedicine\#.U34OBi-YFyd. Accessed May 22, 2014.

2. Gros DF, Morland LA, Greene CJ, et al. Delivery of evidence-based psychotherapy via video telehealth. J Psychopathol Behav Assess. 2013;35(4):506-521.

3. Kessler RC, Aguilar-Gaxiola S, Alonso J, et al. [The global burden of mental disorders: an update from the WHO world mental health (WMH) surveys]. Epidemiol Psichiatr Soc. 2009;18(1):23-33. Italian.

4. Mathers C, Fat DM, Boerma J. The global burden of disease: 2004 update. Geneva, Switzerland: World Health Organization; 2008. Available from: http://www.who.int/healthinfo/global_burden_disease/ GBD_report_2004update_full.pdf. Accessed August 7, 2014. 
5. Kessler RC, Berglund P, Demler O, et al; National Comorbidity Survey Replication. The epidemiology of major depressive disorder: Results from the National Comorbidity Survey Replication (NCS-R). JAMA. 2003;289(23):3095-3105.

6. Kessler RC, Demler O, Frank RG, et al. Prevalence and treatment of mental disorders, 1990 to 2003. N Engl J Med. 2005;352(24):2515-2523.

7. Endicott J. Barriers to seeking treatment for major depression. Depress Anxiety. 1996;4(6):273-278.

8. Wierzbicki M, Pekarik G. A meta-analysis of psychotherapy dropout. Prof Psychol Res Pr. 1993;24(2):190-195.

9. Choi NG, Kunik ME, Wilson N. Mental health service use among depressed, low-income homebound middle-aged and older adults. $J$ Aging Health. 2013;25(4):638-655.

10. Dobkin RD, Menza M, Allen LA, et al. Telephone-based cognitivebehavioral therapy for depression in Parkinson disease. $J$ Geriatr Psychiatry Neurol. 2011;24(4):206-214.

11. Dobkin RD, Rubino JT, Friedman J, Allen LA, Gara MA, Menza M. Barriers to mental health care utilization in Parkinson's disease. J Geriatr Psychiatry Neurol. 2013;26(2):105-116.

12. Weintraub D, Moberg PJ, Duda JE, et al. Recognition and treatment of depression in Parkinson's disease. J Geriatr Psychiatry Neurol. 2003;16(3):178-183

13. Hollon SD, Muñoz RF, Barlow DH, et al. Psychosocial intervention development for the prevention and treatment of depression: promoting innovation and increasing access. Biol Psychiatry. 2002;52(6): 610-630.

14. Collins KA, Westra HA, Dozois DJ, Burns DD. Gaps in accessing treatment for anxiety and depression: challenges for the delivery of care Clin Psychol Rev. 2004;24(5):583-616.

15. Rural Assistance Center. Health professional shortage areas: mental health, designated populations. Available from: http://www.raconline. org/racmaps/mapfiles/hpsa_mental.png. Accessed May 5, 2014.

16. Richards JM, Gottfredson GD. Geographic distribution of US psychologists: a human ecological analysis. Am Psychol. 1978;33(1):1-9.

17. Bull CN, Krout JA, Rathbone-McCuan E, Shreffler MJ. Access and issues of equity in remote/rural areas. J Rural Health. 2001;17(4):356-359.

18. Jameson JP, Blank MB. The role of clinical psychology in rural mental health services: defining problems and developing solutions. Clin Psychol Sci Pract. 2007;14(3):283-298.

19. Marsh L. Neuropsychiatric issues in advancing PD: clinical spectrum and resulting QOL implications. In: Proceedings from the World Parkinson's Congress, September 28-October 1, 2010. Glasgow, Scotland, UK. 2010;25:S570-S570.

20. Quality of Life Research Unit. The quality of life model. Available from: http://www.utoronto.ca/qol/qol_model.htm. Accessed May 5, 2014.

21. Bowling A. What things are important in people's lives? A survey of the public's judgements to inform scales of health related quality of life. Soc Sci Med. 1995;41(10):1447-1462.

22. Mendlowicz MV, Stein MB. Quality of life in individuals with anxiety disorders. Am J Psychiatry. 2000;157(5):669-682.

23. Forsaa EB, Larsen JP, Wentzel-Larsen T, Herlofson K, Alves G. Predictors and course of health-related quality of life in Parkinson's disease. Mov Disord. 2008;23(10):1420-1427.

24. Newman MG. Recommendations for a cost-offset model of psychotherapy allocation using generalized anxiety disorder as an example J Consult Clin Psychol. 2000;68(4):549.

25. Mohr DC, Ho J, Duffecy J, et al. Effect of telephone-administered vs face-to-face cognitive behavioral therapy on adherence to therapy and depression outcomes among primary care patients: a randomized trial. JAMA. 2012;307(21):2278-2285.

26. Brenes GA, Miller ME, Williamson JD, et al. A randomized controlled trial of telephone-delivered cognitive-behavioral therapy for late-life anxiety disorders. Am J Geriatr Psychiatry. 2012;20(8):707-716.

27. Ludman EJ, Simon GE, Tutty S, Von Korff M. A randomized trial of telephone psychotherapy and pharmacotherapy for depression: continuation and durability of effects. J Consult Clin Psychol. 2007;75(2):257-266.
28. Simon GE, Ludman EJ, Rutter CM. Incremental benefit and cost of telephone care management and telephone psychotherapy for depression in primary care. Arch Gen Psychiatry. 2009;66(10):1081-1089.

29. Fortney JC, Pyne JM, Mouden SB, et al. Practice-based versus telemedicine-based collaborative care for depression in rural federally qualified health centers: a pragmatic randomized comparative effectiveness trial. Am J Psychiatry. 2013;170(4):414-425.

30. Stiles-Shields C, Kwasny MJ, Cai X, Mohr DC. Therapeutic alliance in face-to-face and telephone-administered cognitive behavioral therapy. J Consult Clin Psychol. 2014;80(2):349-354.

31. Applebaum AJ, DuHamel KN, Winkel G, et al. Therapeutic alliance in telephone-administered cognitive-behavioral therapy for hematopoietic stem cell transplant survivors. J Consult Clin Psychol. 2012;80(5):811-816.

32. Reger MA, Gahm GA. A meta-analysis of the effects of Internet-and computer-based cognitive-behavioral treatments for anxiety. $J$ Clin Psychol. 2009;65(1):53-75.

33. Andersson G, Cuijpers P. Internet-based and other computerized psychological treatments for adult depression: a meta-analysis. Cogn Behav Ther. 2009;38(4):196-205.

34. Christensen H, Reynolds J, Griffiths KM. The use of e-health applications for anxiety and depression in young people: challenges and solutions. Early Interv Psychiatry. 2011;5(1):58-62.

35. Richardson T, Stallard P, Velleman S. Computerised cognitive behavioural therapy for the prevention and treatment of depression and anxiety in children and adolescents: a systematic review. Clin Child Fam Psychol Rev. 2010;13(3):275-290.

36. Leach LS, Christensen H. A systematic review of telephone-based interventions for mental disorders. J Telemed Telecare. 2006;12(3):122-129.

37. Newman MG, Szkodny LE, Llera SJ, Przeworski A. A review of technology-assisted self-help and minimal contact therapies for anxiety and depression: is human contact necessary for therapeutic efficacy? Clin Psychol Rev. 2011;31(1):89-103.

38. Carlbring P, Andersson G. Internet and psychological treatment. How well can they be combined? Comput Human Behav. 2006;22(3): $545-553$.

39. Griffiths KM, Christensen H. Internet-based mental health programs: a powerful tool in the rural medical kit. Aust J Rural Health. 2007; 15(2):81-87.

40. Paul CL, Carey ML, Sanson-Fisher RW, Houlcroft LE, Turon HE. The impact of web-based approaches on psychosocial health in chronic physical and mental health conditions. Health Educ Res. 2013;28(3): 450-471.

41. Postel MG, de Haan HA, De Jong CA. E-therapy for mental health problems: a systematic review. Telemed J E Health. 2008;14(7):707-714.

42. Proudfoot JG. Computer-based treatment for anxiety and depression: Is it feasible? Is it effective? Neurosci Biobehav Rev. 2004;28(3):353-363.

43. Spek V, Cuijpers P, Nyklícek I, Riper H, Keyzer J, Pop V. Internet-based cognitive behaviour therapy for symptoms of depression and anxiety: a meta-analysis. Psychol Med. 2007;37(3):319-328.

44. Catalan J, Gath D, Anastasiades P, Bond S, Day A, Hall L. Evaluation of a brief psychological treatment for emotional disorders in primary care. Psychol Med. 1991;21(4):1013-1018.

45. Katon W, Von Korff M, Lin E, et al. Stepped collaborative care for primary care patients with persistent symptoms of depression: a randomized trial. Arch Gen Psychiatry. 1999;56(12):1109-1115.

46. Rost K, Nutting P, Smith J, Werner J, Duan N. Improving depression outcomes in community primary care practice. J Gen Intern Med. 2001;16(3):143-149.

47. Wells KB, Sherbourne C, Schoenbaum M, et al. Impact of disseminating quality improvement programs for depression in managed primary care: a randomized controlled trial. JAMA. 2000;283(2):212-220.

48. Bruce ML, Ten Have TR, Reynolds CF III, et al. Reducing suicidal ideation and depressive symptoms in depressed older primary care patients: a randomized controlled trial. JAMA. 2004;291(9):1081-1091.

49. Unützer J, Katon W, Callahan CM, et al. Collaborative care management of late-life depression in the primary care setting: a randomized controlled trial. JAMA. 2002;288(22):2836-2845. 
50. Williams JW Jr, Rost K, Dietrich AJ, Ciotti MC, Zyzanski SJ, Cornell J. Primary care physicians' approach to depressive disorders: effects of physician specialty and practice structure. Arch Fam Med. 1999;8(1):58-67.

51. Fortney JC, Pyne JM, Edlund MJ, et al. A randomized trial of telemedicine-based collaborative care for depression. J Gen Intern Med. 2007;22(8):1086-1093.

52. Griffiths KM, Christensen H. Review of randomised controlled trials of Internet interventions for mental disorders and related conditions. Clin Psychol. 2006;10(1):16-29.

53. Carlbring $\mathrm{P}, \mathrm{Bohman} \mathrm{S}$, Brunt $\mathrm{S}$, et al. Remote treatment of panic disorder: a randomized trial of Internet-based cognitive behavior therapy supplemented with telephone calls. Am J Psychiatry. 2006;163(12):2119-2125.

54. Cavanagh K, Shapiro D, Berg S, Swain S, Barkham M, Proudfoot J. The effectiveness of computerized cognitive behavioural therapy in routine care. Br J Clin Psychol. 2006;45(4):499-514.

55. Marks IM, Mataix-Cols D, Kenwright M, Cameron R, Hirsch S, Gega L. Pragmatic evaluation of computer-aided self-help for anxiety and depression. Br J Psychiatry. 2003;183:57-65.

56. Wright JH, Wright AS, Albano AM, et al. Computer-assisted cognitive therapy for depression: maintaining efficacy while reducing therapist time. Am J Psychiatry. 2005;162(6):1158-1164.

57. Wright JH, Wright AS, Salmon P, et al. Development and initial testing of a multimedia program for computer-assisted cognitive therapy. Am J Psychother. 2002;56(1):76-86.

58. Palmqvist B, Carlbring P, Andersson G. Internet-delivered treatments with or without therapist input: Does the therapist factor have implications for efficacy and cost? Expert Rev Pharmacoecon Outcomes. 2007;7(3):291-297.

59. Sethi S. Treating youth depression and anxiety: a randomised controlled trial examining the efficacy of computerised versus face-to-face cognitive behaviour therapy. Aust Psychol. 2013;48(4):249-257.

60. Baron KG, Corden M, Jin L, Mohr DC. Impact of psychotherapy on insomnia symptoms in patients with depression and multiple sclerosis. J Behav Med. 2011;34(2):92-101.

61. Mohr DC, Hart S, Vella L. Reduction in disability in a randomized controlled trial of telephone-administered cognitive-behavioral therapy. Health Psychol. 2007;26(5):554-563.

62. Cosio D, Jin L, Siddique J, Mohr DC. The effect of telephone-administered cognitive-behavioral therapy on quality of life among patients with multiple sclerosis. Ann Behav Med. 2011;41(2):227-234.

63. Mohr DC, Likosky W, Bertagnolli A, et al. Telephone-administered cognitive-behavioral therapy for the treatment of depressive symptoms in multiple sclerosis. J Consult Clin Psychol. 2000;68(2):356-361.

64. Bombardier CH, Ehde DM, Gibbons LE, et al. Telephone-based physical activity counseling for major depression in people with multiple sclerosis. J Consult Clin Psychol. 2013;81(1):89-99.

65. Dorstyn D, Mathias JL, Denson LA. Psychosocial outcomes of telephone-based counseling for adults with an acquired physical disability: a meta-analysis. Rehabil Psychol. 2011;56(1):1-14.

66. Motl RW, Dlugonski D, Wojcicki TR, McAuley E, Mohr DC. Internet intervention for increasing physical activity in persons with multiple sclerosis. Mult Scler. 2011;17(1):116-128.

67. Dlugonski D, Motl RW, Mohr DC, Sandroff BM. Internet-delivered behavioral intervention to increase physical activity in persons with multiple sclerosis: sustainability and secondary outcomes. Psychol Health Med. 2012;17(6):636-651.

68. Grossman P, Kappos L, Gensicke H, et al. MS quality of life, depression, and fatigue improve after mindfulness training: a randomized trial. Neurology. 2010;75(13):1141-1149.

69. Suh Y, Motl RW, Mohr DC. Physical activity, disability, and mood in the early stage of multiple sclerosis. Disabil Health J. 2010;3(2):93-98.

70. Mohr DC, Hart SL, Julian L, et al. Telephone-administered psychotherapy for depression. Arch Gen Psychiatry. 2005;62(9):1007-1014.

71. Veazey C, Cook KF, Stanley M, LaiEC, Kunik ME. Telephone-administered cognitive behavioral therapy: a case study of anxiety and depression in Parkinson's disease. J Clin Psychol Med Settings. 2009;16(3):243-253.
72. Ransom D, Heckman TG, Anderson T, Garske J, Holroyd K, Basta T. Telephone-delivered, interpersonal psychotherapy for HIV-infected rural persons with depression: a pilot trial. Psychiatr Serv. 2008;59(8): 871-877.

73. Himelhoch S, Medoff D, Maxfield J, et al. Telephone based cognitive behavioral therapy targeting major depression among urban dwelling, low income people living with HIV/AIDS: results of a randomized controlled trial. AIDS Behav. 2013;17(8):2756-2764.

74. Gentry S, van-Velthoven MHMMT, Tudor Car L, Car J. Telephone delivered interventions for reducing morbidity and mortality in people with HIV infection. Cochrane Database of Systematic Reviews. 2013;5:CD009189.

75. Piette JD, Richardson C, Himle J, et al. A randomized trial of telephonic counseling plus walking for depressed diabetes patients. Med Care. 2011;49(7):641-648.

76. Katon WJ, Lin EH, Von Korff M, et al. Collaborative care for patients with depression and chronic illnesses. $N$ Engl J Med. 2010;363(27): 2611-2620.

77. Rollman BL, Belnap BH, LeMenager MS, et al. Telephone-delivered collaborative care for treating post-CABG depression: a randomized controlled trial. JAMA. 2009;302(19):2095-2103.

78. Wade SL, Carey J, Wolfe CR. An online family intervention to reduce parental distress following pediatric brain injury. J Consult Clin Psychol. 2006;74(3):445-454.

79. Wade SL, Walz NC, Carey J, et al. A randomized trial of teen online problem solving: efficacy in improving caregiver outcomes after brain injury. Health Psychol. 2012;31(6):767-776.

80. Winter L, Gitlin LN. Evaluation of a telephone-based support group intervention for female caregivers of community-dwelling individuals with dementia. Am J Alzheimers Dis Other Demen. 2006;21(6):391-397.

81. Glueckauf RL, Davis WS, Willis F, et al. Telephone-based, cognitivebehavioral therapy for African American dementia caregivers with depression: initial findings. Rehabil Psychol. 2012;57(2):124-139.

82. Glueckauf RL, Jeffers SB, Sharma D, et al. Telephone-based cognitivebehavioral intervention for distressed rural dementia caregivers: initial findings. Clin Gerontol. 2007;31(1):21-41.

83. DuHamel KN, Mosher CE, Winkel G, et al. Randomized clinical trial of telephone-administered cognitive-behavioral therapy to reduce posttraumatic stress disorder and distress symptoms after hematopoietic stem-cell transplantation. J Clin Oncol. 2010;28(23):3754-3761.

84. Forducey PG, Glueckauf RL, Bergquist TF, Maheu MM, Yutsis M. Telehealth for persons with severe functional disabilities and their caregivers: facilitating self-care management in the home setting. Psychol Serv. 2012;9(2):144-162.

85. American Diabetes Association. Standards of medical care in diabetes - 2009. Diabetes Care. 2009;32 Suppl 1:13-61.

86. Hansen NB, Lambert MJ, Forman EM. The psychotherapy doseresponse effect and its implications for treatment delivery services. Clin Psychol Sci Pract. 2002;9(3):329-343.

87. Klerman GL, Weissman MM, Rounsaville BJ, Chevron ES. Interpersonal Psychotherapy for Depression: A Brief, Focused, Specific Strategy. 1st ed. Lanham, MD, USA: Jason Aronson Inc.; 1994.

88. Andersson G, Bergstrom J, Hollandare F, Carlbring P, Kaldo V, Ekselius L. Internet-based self-help for depression: randomised controlled trial. Br J Psychiatry. 2005;187:456-461.

89. Tutty S, Spangler DL, Poppleton LE, Ludman EJ, Simon GE. Evaluating the effectiveness of cognitive-behavioral teletherapy in depressed adults. Behav Ther. 2010;41(2):229-236.

90. Proudfoot J, Goldberg D, Mann A, Everitt B, Marks I, Gray JA. Computerized, interactive, multimedia cognitive-behavioural program for anxiety and depression in general practice. Psychol Med. 2003;33(2):217-227.

91. Hunkeler EM, Hargreaves WA, Fireman B, et al. A web-delivered care management and patient self-management program for recurrent depression: a randomized trial. Psychiatr Serv. 2012;63(11): 1063-1071. 
92. Dwight-Johnson M, Aisenberg E, Golinelli D, Hong S, O’Brien M, Ludman E. Telephone-based cognitive-behavioral therapy for Latino patients living in rural areas: a randomized pilot study. Psychiatr Serv. 2011;62(8):936-942.

93. Ruskin PE, Silver-Aylaian M, Kling MA, et al. Treatment outcomes in depression: comparison of remote treatment through telepsychiatry to in-person treatment. Am J Psychiatry. 2004;161(8):1471-1476.

94. Moreno FA, Chong J, Dumbauld J, Humke M, Byreddy S. Use of standard webcam and Internet equipment for telepsychiatry treatment of depression among underserved Hispanics. Psychiatr Serv. 2012;63(12):1213-1217.

95. Marin H, Escobar JI, Vega WA. Mental illness in Hispanics: a review of the literature. FOCUS. 2006;4(1):23-37.

96. Pearson B, Katz SE, Soucie V, et al. Evidence-based care for depression in Maine: dissemination of the Kaiser Permanente nurse telecare program. Psychiatr Q. 2003;74(1):91-102.

97. Choi NG, Marti CN, Bruce ML, Hegel MT. Depression in homebound older adults: problem-solving therapy and personal and social resourcefulness. Behav Ther. 2013;44(3):489-500.

98. Andersson G, Carlbring P, Holmström A, et al. Internet-based self-help with therapist feedback and in vivo group exposure for social phobia: a randomized controlled trial. J Consult Clin Psychol. 2006;74(4): 677-686.

99. Klein B, Richards JC, Austin DW. Efficacy of Internet therapy for panic disorder. J Behav Ther Exp Psychiatry. 2006;37(3):213-238.

100. Hecker JE, Losee MC, Roberson-Nay R, Maki K. Mastery of your anxiety and panic and brief therapist contact in the treatment of panic disorder. J Anxiety Disord. 2004;18(2):111-126.

101. Schneider AJ, Mataix-Cols D, Marks IM, Bachofen M. Internet-guided self-help with or without exposure therapy for phobic and panic disorders. Psychother Psychosom. 2005;74(3):154-164.

102. Bergström J, Andersson G, Karlsson A, et al. An open study of the effectiveness of internet treatment for panic disorder delivered in a psychiatric setting. Nord J Psychiatry. 2009;63(1):44-50.

103. Carlbring P, Westling BE, Ljungstrand P, Ekselius L, Andersson G. Treatment of panic disorder via the Internet: a randomized trial of a self-help program. Behav Ther. 2001;32(4):751-764.

104. Carlbring P, Ekselius L, Andersson G. Treatment of panic disorder via the Internet: a randomized trial of CBT vs applied relaxation. J Behav Ther Exp Psychiatry. 2003;34(2):129-140.

105. Carlbring P, Nilsson-Ihrfelt E, Waara J, et al. Treatment of panic disorder: live therapy vs self-help via the Internet. Behav Res Ther. 2005;43(10):1321-1333.

106. Sloan DM, Gallagher MW, Feinstein BA, Lee DJ, Pruneau GM. Efficacy of telehealth treatments for posttraumatic stress-related symptoms: a meta-analysis. Cogn Behav Ther. 2011;40(2):111-125.

107. Gros DF, Yoder M, Tuerk PW, Lozano BE, Acierno R. Exposure therapy for PTSD delivered to veterans via telehealth: predictors of treatment completion and outcome and comparison to treatment delivered in person. Behav Ther. 2011;42(2):276-283.

108. Litz B, Engel C, Bryant R, Papa A. A randomized, controlled proof-ofconcept trial of an internet-based, therapist-assisted self-management treatment for posttraumatic stress disorder. Am J Psychiatry. 2007;164(11):1676-1684.
109. Frueh BC, Monnier J, Yim E, Grubaugh AL, Hamner MB, Knapp RG A randomized trial of telepsychiatry for post-traumatic stress disorder. J Telemed Telecare. 2007;13(3):142-147.

110. Gros DF, Veronee K, Strachan M, Ruggiero KJ, Acierno R. Managing suicidality in home-based telehealth. $J$ Telemed Telecare. 2011;17(6):332-335.

111. Kazdin AE, Blase SL. Rebooting psychotherapy research and practice to reduce the burden of mental illness. Perspect Psychol Sci. 2011;6(1): 21-37.

112. Shapiro DA, Cavanagh K, Lomas H. Geographic inequity in the availability of cognitive behavioural therapy in England and Wales. Behav Cogn Psychother. 2003;31(2):185-192.

113. Shedler J. The efficacy of psychodynamic psychotherapy. Am Psychol. 2010;65(2):98-109.

114. Knaevelsrud C, Maercker A. Internet-based treatment for PTSD reduces distress and facilitates the development of a strong therapeutic alliance: a randomized controlled clinical trial. BMC Psychiatry. 2007;7(13):1-10

115. Wangberg SC, Gammon D, Spitznogle K. In the eyes of the beholder: exploring psychologists' attitudes towards and use of e-therapy in Norway. Cyberpsychol Behav. 2007;10(3):418-423.

116. Mora L, Nevid J, Chaplin W. Psychologist treatment recommendations for Internet-based therapeutic interventions. Comput Human Behav. 2008;24(6):3052-3062.

117. Mitchell JE, Crosby RD, Wonderlich SA, et al. A randomized trial comparing the efficacy of cognitive-behavioral therapy for bulimia nervosa delivered via telemedicine versus face-to-face. Behav Res Ther. 2008;46(5):581-592.

118. Austen S, McGrath M. Attitudes to the use of videoconferencing in general and specialist psychiatric services. $J$ Telemed Telecare. 2006;12(3):146-150.

119. Rees CS, Stone S. Therapeutic alliance in face-to-face versus videoconferenced psychotherapy. Prof Psychol Res Pr. 2005;36(6):649-653.

120. Ertelt TW, Crosby RD, Marino JM, Mitchell JE, Lancaster K, Crow SJ. Therapeutic factors affecting the cognitive behavioral treatment of bulimia nervosa via telemedicine versus face-to-face delivery. Int $J$ Eat Disord. 2011;44(8):687-691.

121. Achey M, Aldred JL, Aljehani N, et al. The past, present, and future of telemedicine for Parkinson's disease. Mov Disord 2014;29(7):871-883.

122. Godleski L, Cervone D, Vogel D, Rooney M. Home telemental health implementation and outcomes using electronic messaging. J Telemed Telecare. 2012;18(1):17-19.

123. O’Reilly R, Bishop J, Maddox K, Hutchinson L, Fisman M, Takhar J. Is telepsychiatry equivalent to face-to-face psychiatry? Results from a randomized controlled equivalence trial. Psychiatr Serv. 2007;58(6): 836-843.

124. Cryer L, Shannon SB, Van Amsterdam M, Leff B. Costs for 'hospital at home' patients were 19 percent lower, with equal or better outcomes compared to similar inpatients. Health Aff (Millwood). 2012;31(6): $1237-1243$.
Smart Homecare Technology and TeleHealth

\section{Publish your work in this journal}

Smart Homecare Technology and TeleHealth is an international, peer-reviewed, open access online journal publishing original research, reviews, editorials and commentaries on the application of technology to support people and patients at home and in assisted living centers to optimize healthcare and management resources. Specific topics in the journal include: Development and application of

\section{Dovepress}

devices within the home and embedded in appliances; Healthcare provider communication and education tools; and drug ordering and adherence. The manuscript management system is completely online and includes a very quick and fair peer-review system, which is all easy to use. Visit http://www.dovepress.com/ testimonials.php to read real quotes from published authors. 